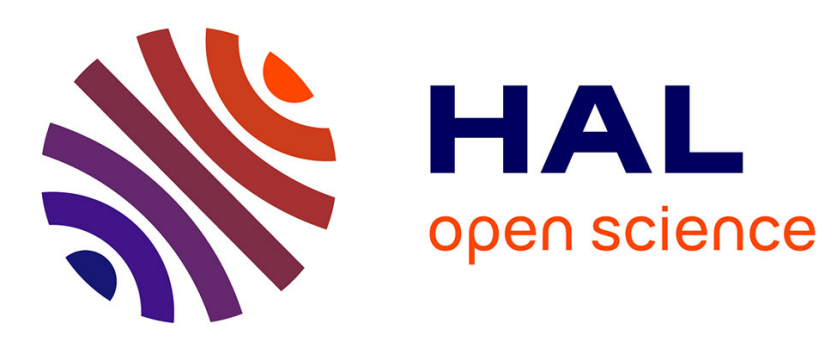

\title{
Asymptotic derivation of a linear plate model for soft ferromagnetic materials
}

Giuseppe Geymonat, Françoise Krasucki, Michele Serpilli

\section{To cite this version:}

Giuseppe Geymonat, Françoise Krasucki, Michele Serpilli. Asymptotic derivation of a linear plate model for soft ferromagnetic materials. Chinese Annals of Mathematics - Series B, 2018, 39 (3), pp.451-460. 10.1007/s11401-018-0077-5 . hal-01817337

\section{HAL Id: hal-01817337 \\ https://hal.science/hal-01817337}

Submitted on 17 Jun 2018

HAL is a multi-disciplinary open access archive for the deposit and dissemination of scientific research documents, whether they are published or not. The documents may come from teaching and research institutions in France or abroad, or from public or private research centers.
L'archive ouverte pluridisciplinaire HAL, est destinée au dépôt et à la diffusion de documents scientifiques de niveau recherche, publiés ou non, émanant des établissements d'enseignement et de recherche français ou étrangers, des laboratoires publics ou privés. 


\title{
ASYMPTOTIC DERIVATION OF A LINEAR PLATE MODEL FOR SOFT FERROMAGNETIC MATERIALS
}

\author{
Giuseppe GEYMONAT ${ }^{1} \quad$ Françoise KRASUCKI ${ }^{2} \quad$ Michele SERPILLI $^{3}$
}

\begin{abstract}
We apply the asymptotic expansion method by P.G. Ciarlet to obtain a Kirchhoff-Love-type plate model for a linear soft ferromagnetic material. We also give a mathematical justification of the obtained model by means of a strong convergence result.
\end{abstract}

Keywords Asymptotic methods, Plates, Magnetoelasticity

2010 MR Subject Classification 74F15, 74K20

\section{Introduction}

The use of the framework of continuum mechanics for the study of the influence of electromagnetic effects on solids has been largely stimulated by Truesdell and Toupin [14]. Their research on the coupling between the mechanical and magnetic responses of magnetoelastic solids was the first of a long list (without any attempt to be exhaustive, see, e.g. Brown [1] Tiersten [13], Maugin and Eringen [6], Pao and Yeh [12], Moon [9], Maugin [8], Dorfmann and Ogden [4], Kankanala and Triantafyllidis [5], ...). An important stimulus for the development of these researches was the study of the magnetoelastic buckling problem. Indeed a plate, made of a magnetoelastic material, subject to a transverse magnetic field, buckles when the magnetic field attains a critical value; see also [5, 3], for a general analysis of the buckling of some magnetoelastic structures. Following a pioneering experimental and theoretical research of Moon and Pao, the first rigorous attempt to analyze this problem is due to Pao and Yeh [12]. Maugin and Goudjo [7] have considered a plate model with particular attention on the regularity of the boundary. More recently, in the case linear soft magnetoelastic materials, Zhou and Zheng $[15,16,17]$ have revisited the subject by adapting the usual Kirchhoff-Love and von Kármán models only modifying the equivalent transverse force with the addition of the magnetic effects.

The paper is organized as follows. In Sect. 2, we briefly recall the governing equilibrium equations of magnetoelasticity and, then, in Sect. 3, we state the problem on a variable domain

\footnotetext{
Manuscript received

${ }^{1}$ Laboratoire de Mécanique des Solides, École Polytechnique, CNRS, Universit Paris-Saclay, 91128 Palaiseau, France

E-mail:giuseppe.geymonat@polytechnique.edu

${ }^{2}$ Institut Montpelli04erain Alexander Grothendieck, UMR-CNRS 5149, Université de Montpellier

Place Eugène Bataillon, 34095 Montpellier Cedex 5, France

E-mail:francoise.krasucki@umontpellier.fr

${ }^{3}$ Department of Civil and Building Engineering, and Architecture,

Polytechnic University of Marche, Via Brecce Bianche, 60131 Ancona, Italy

E-mail:m.serpilli@univpm.it
} 
assuming that the magnetic forces are given. In order to apply the Ciarlet's method (see, e.g., [2]), we must at first prove that the magnetic forces give rise to a linear and continuous form. This can be achieved under suitable assumptions on the magnetic forces, see lemma 3.1. In Sect. 4, we introduce the usual scaling on the mechanical quantities and we scale the magnetic quantities in such a way that the Gauss' Law (2.1) is conserved. Using the classical change of variables, we deduce the scaled equations. Then we can apply the asymptotic methods, following [2], to obtain the limit problem and the strong convergence result. It is interesting to remark that in the simplest situation of a transversal magnetic field, we recover the Kirchhoff-Love model of Zhou and Zheng, which is, hence, completely justified.

\section{Governing equations of magnetoelasticity for linear soft ferromag- netic materials}

In the sequel, Greek indices range in the set $\{1,2\}$, Latin indices range in the set $\{1,2,3\}$, and the Einstein's summation convention with respect to the repeated indices is adopted. Let us consider a three-dimensional

Euclidean space identified by $\mathbb{R}^{3}$ and such that the three vectors $\mathbf{e}_{i}$ form an orthonormal basis. We introduce the following notations for the vector product: $\mathbf{a} \wedge \mathbf{b}=a_{i} \mathbf{e}_{i} \wedge b_{j} \mathbf{e}_{j}=a_{i} b_{j} \epsilon_{i j k} \mathbf{e}_{k}$, for all vectors $\mathbf{a}=\left(a_{i}\right) \in \mathbb{R}^{3}$ and $\mathbf{b}=\left(b_{i}\right) \in \mathbb{R}^{3}$, where $\epsilon_{i j k}$ denotes the alternator Ricci's symbol.

When a magnetizable, deformable elastic solid $\Omega$ is placed in a magnetic field, magnetic moments are induced inside the body. The action of the external magnetic induction $\mathbf{B}_{0}$ manifests itself in magnetization $\mathbf{M}$ (magnetic moment per unit volume). Within the body, the magnetic induction $\mathbf{B}$ is not necessarily equal to $\mathbf{B}_{0}$. The induced magnetization $\mathbf{M}=\left(M_{i}\right)$ is related to $\mathbf{B}=\left(B_{i}\right)$ by $\mathbf{B}=\mu_{0}(\mathbf{H}+\mathbf{M})$, where $\mathbf{H}=\left(H_{i}\right)$ is called magnetic intensity and $\mu_{0}$ is the magnetic permeability of vacuum. Generally, we have $\mathbf{H}=\mathbf{H}(\mathbf{M})$, but, in the sequel, we restrict our attention to a class of linear isotropic magnetoelastic materials called soft ferromagnetic materials, which are characterized by the fact that their local average magnetization becomes zero when the external field is set to zero. In this particular case, the hysteresis loops are narrow and the influence of induced currents is small in comparison with the effect of magnetization. Therefore, it is possible to use the quasi-static approximation, i.e., the equations of magneto-statics:

$$
\left\{\begin{array}{lll}
\partial_{i} B_{i}=0 & \text { in } \Omega, & \text { (Gauss' Law) } \\
\epsilon_{k i j} \partial_{j} H_{i}=0 & \text { in } \Omega . & \text { (Ampère's Law) }
\end{array}\right.
$$

The magnetic constitutive law takes the following linear form

$$
\mathbf{M}=\chi \mathbf{H} \text { or } \mathbf{B}=\mu_{0} \mu_{r} \mathbf{H},
$$

where $\chi$ represents the magnetic susceptibility and $\mu_{r}:=\chi+1$ is the relative magnetic permeability. For linear soft ferromagnetic materials, such as steels, iron, cobalt and various alloys, the relative permeability is very large, $\mu_{r}$ or $\chi=10^{2} \sim 10^{5}$.

In this work we use the model proposed by Brown [1] where the action of the magnetic field is given by a magnetic body force (per unit volume) $\mathbf{f}^{m}=\left(f_{i}^{m}\right)$ and a magnetic body couple (per unit volume) $\mathbf{I}^{m}=\left(I_{i}^{m}\right)$ :

$$
\mathbf{f}^{m}=\mu_{0}(\boldsymbol{\nabla} \mathbf{H}) \mathbf{M} \text { and } \mathbf{I}^{m}=\mu_{0} \mathbf{M} \wedge \mathbf{H}
$$


i.e. component-wise, $f_{i}^{m}=\mu_{0} M_{j} \partial_{j} H_{i}$ and $I_{i}^{m}=\mu_{0} \epsilon_{k j i} M_{k} H_{j}$, This choice of the action of the magnetic field is sometimes called the dipole model of microcurrents and has been used in particular by Pao-Yeh for soft ferromagnetic elastic solids [12]. Using the Gauss' law and the Ampère's law, the magnetic body force $\mathbf{f}^{m}$ can also be written as the divergence of a second order tensor $\mathbf{T}^{m}=\left(T_{i j}^{m}\right)$, the so-called Maxwell's stress tensor

$$
\mathbf{f}^{m}=\operatorname{div} \mathbf{T}^{m},
$$

or, component-wise, $f_{j}^{m}=\partial_{i} T_{i j}^{m}$ with $T_{i j}^{m}:=B_{i} H_{j}-\frac{1}{2} \mu_{0} H^{2} \delta_{i j}$ and $H^{2}:=H_{k} H_{k}$. As pointed out by [1], other different choices of the Maxwell's stress tensor are possible and, indeed, they depend on the choice of the Helmomtz free energy; see in particular [5], for a clear explanation of the influence of the choice of the arguments in the free energy on the Maxwell stress, magnetic body forces and traction boundary conditions.

Considering the expressions above, in the absence of electric field, charge distribution and conduction current, the mechanical governing equations defined in a magnetized body $\Omega$ can be expressed by

$$
\begin{cases}\partial_{i} t_{i j}+f_{j}^{m}=0 & \text { in } \Omega, \\ t_{i j}-t_{j i}+\mu_{0}\left(M_{i} H_{j}-M_{j} H_{i}\right)=0 & \text { in } \Omega, \\ t_{i j} n_{i}=\frac{1}{2} \mu_{0}\left(M_{n}\right)^{2} n_{j} & \text { in } \Xi,\end{cases}
$$

where $t_{i j}$ is the non-symmetric total stress tensor, $\left(n_{i}\right)$ represents the unit normal vector to the boundary $\Xi \subset \partial \Omega$ and $M_{n}:=M_{i} n_{i}$ is the normal surface boundary magnetization. The nonsymmetry of the total stress tensor is due to the presence of a magnetic body couple. In order to simplify the model, we neglect magnetostriction and piezostrictive terms in the constitutive laws and we consider an isotropic linear elastic material. Thus, we obtain that

$$
t_{i j}=\sigma_{i j}+\mu_{0} M_{i} H_{j}, \text { with } \sigma_{i j}=\lambda e_{p p} \delta_{i j}+2 \mu e_{i j},
$$

where $\sigma_{i j}$ denotes the symmetric Cauchy stress tensor, associated with the linearized strain tensor $e_{i j}=\frac{1}{2}\left(\partial_{i} u_{j}+\partial_{j} u_{i}\right)$, being $\left(u_{i}\right)$ the displacement field, through the classical Lamé's constitutive equations. The presence of the term $\mu_{0} M_{i} H_{j}$ in the decomposition of the total stress always follows from the form of the Helmoltz free energy (see [1] and [5]).

In the Pao-Yeh's case of soft ferromagnetic materials, thanks to (2.2), the magnetic body couple $\mathbf{I}^{m}=\mu_{0} \chi \mathbf{H} \wedge \mathbf{H}=\mathbf{0}$, and, hence, the stress tensor $t_{i j}$ becomes symmetric, i.e., $t_{i j}=t_{j i}$. Moreover, substituting the expression of the Maxwell's stress tensor into the divergence relation (2.3) and using (2.1), we can find an alternative form of the magnetic body force:

$$
\mathbf{f}^{m}=\mu_{0}(\nabla \mathbf{H}) \mathbf{M}=\frac{\mu_{0} \chi}{2} \nabla\left(H^{2}\right) .
$$

In the sequel, we will focus our attention to the reduced mechanical model arising from the use of the asymptotic methods, assuming that the magnetization $\mathbf{M}$ and the magnetic intensity $\mathbf{H}$ are a given external magnetic source.

\section{Position of the problem}

Let $\omega \subset \mathbb{R}^{2}$ denote a smooth domain in the plane spanned by vectors $\mathbf{e}_{\alpha}$, with boundary $\gamma ; \gamma_{0} \subset \gamma$ is a measurable subset of $\gamma$ with strictly positive length measure; $\gamma_{1}:=\gamma \backslash \gamma_{0}$ is the 
complement of $\gamma_{0}$ with respect to $\gamma$; finally, $0<\varepsilon<1$ is a dimensionless small real parameter which shall tend to zero. For each $\varepsilon$, we define

$$
\begin{array}{ll}
\Omega^{\varepsilon}:=\omega \times\left(-h^{\varepsilon}, h^{\varepsilon}\right), & \Gamma^{\varepsilon}:=\gamma \times\left(-h^{\varepsilon}, h^{\varepsilon}\right) \\
\Gamma_{0}^{\varepsilon}:=\gamma_{0} \times\left(-h^{\varepsilon}, h^{\varepsilon}\right), & \Gamma_{ \pm}^{\varepsilon}:=\omega \times\left\{ \pm h^{\varepsilon}\right\},
\end{array}
$$

with $h^{\varepsilon}>0$. Hence the boundary $\partial \Omega^{\varepsilon}$ of $\Omega^{\varepsilon}$ is partitioned into the lateral surface $\Gamma^{\varepsilon}$ and the upper and lower faces $\Gamma_{+}^{\varepsilon}$ and $\Gamma_{-}^{\varepsilon}$; the lateral surface is itself partitioned as $\Gamma^{\varepsilon}=\Gamma_{0}^{\varepsilon} \cup \Gamma_{1}^{\varepsilon}$, with $\Gamma_{1}^{\varepsilon}:=\gamma_{1} \times\left(-h^{\varepsilon}, h^{\varepsilon}\right)$. Moreover, we let $\widehat{\Gamma}^{\varepsilon}:=\Gamma_{ \pm}^{\varepsilon} \cup \Gamma_{1}^{\varepsilon}=\partial \Omega^{\varepsilon} \backslash \Gamma_{0}^{\varepsilon}$, the complement of $\Gamma_{0}^{\varepsilon}$ with respect to $\partial \Omega^{\varepsilon}$.

We assume that $\Omega^{\varepsilon}$ is constituted by a homogeneous isotropic linear soft ferromagnetic material, whose constitutive law is given in (2.5). We suppose that the Lamé's coefficients satisfy the classical positivity properties. The plate is clamped on $\Gamma_{0}^{\varepsilon}$, so that $\mathbf{u}^{\varepsilon}=\mathbf{0}$, and, for simplicity, we consider that no mechanical charges are applied to the body. The only source terms are given by $M_{i}^{\varepsilon}$ and $H_{i}^{\varepsilon}$.

Let $V\left(\Omega^{\varepsilon}\right):=\left\{\mathbf{v}^{\varepsilon} \in H^{1}\left(\Omega^{\varepsilon} ; \mathbb{R}^{3}\right) ; \mathbf{v}^{\varepsilon}=\mathbf{0}\right.$ on $\left.\Gamma_{0}^{\varepsilon}\right\}$ be the functional space of admissible displacements. The variational formulation of problem (2.4), defined on the variable domain $\Omega^{\varepsilon}$, takes the following form

$$
\left\{\begin{array}{l}
\text { Find } \mathbf{u}^{\varepsilon}=\left(u_{i}^{\varepsilon}\right) \in V\left(\Omega^{\varepsilon}\right) \text { such that } \\
A^{\varepsilon}\left(\mathbf{u}^{\varepsilon}, \mathbf{v}^{\varepsilon}\right)=L^{\varepsilon}\left(\mathbf{v}^{\varepsilon}\right), \text { for all } \mathbf{v}^{\varepsilon}=\left(v_{i}^{\varepsilon}\right) \in V\left(\Omega^{\varepsilon}\right),
\end{array}\right.
$$

where the bilinear form $A^{\varepsilon}(\cdot, \cdot)$ and the linear form $L^{\varepsilon}(\cdot)$ are, respectively, defined by

$$
A^{\varepsilon}\left(\mathbf{u}^{\varepsilon}, \mathbf{v}^{\varepsilon}\right):=\int_{\Omega^{\varepsilon}} t_{i j}^{\varepsilon} e_{i j}^{\varepsilon}\left(\mathbf{v}^{\varepsilon}\right) d x^{\varepsilon}, \quad L^{\varepsilon}\left(\mathbf{v}^{\varepsilon}\right):=\int_{\Omega^{\varepsilon}} \mu_{0} M_{j}^{\varepsilon} \partial_{j}^{\varepsilon} H_{i}^{\varepsilon} v_{i}^{\varepsilon} d x^{\varepsilon}+\int_{\widehat{\Gamma}^{\varepsilon}} \frac{1}{2} \mu_{0}\left(M_{n}^{\varepsilon}\right)^{2} n_{i}^{\varepsilon} v_{i}^{\varepsilon} d \Gamma^{\varepsilon},
$$

with $t_{i j}^{\varepsilon}:=\lambda e_{p p}^{\varepsilon}\left(\mathbf{u}^{\varepsilon}\right) \delta_{i j}+2 \mu e_{i j}^{\varepsilon}\left(\mathbf{u}^{\varepsilon}\right)+\mu_{0} M_{i}^{\varepsilon} H_{j}^{\varepsilon}$.

In order to prove the wellposedness of the problem, by virtue of the Lax-Milgram's lemma, we rewrite (3.1) in an alternative form:

$$
\left\{\begin{array}{l}
\text { Find } \mathbf{u}^{\varepsilon}=\left(u_{i}^{\varepsilon}\right) \in V\left(\Omega^{\varepsilon}\right) \text { such that } \\
\bar{A}^{\varepsilon}\left(\mathbf{u}^{\varepsilon}, \mathbf{v}^{\varepsilon}\right)=\bar{L}^{\varepsilon}\left(\mathbf{v}^{\varepsilon}\right), \text { for all } \mathbf{v}^{\varepsilon}=\left(v_{i}^{\varepsilon}\right) \in V\left(\Omega^{\varepsilon}\right)
\end{array}\right.
$$

where

$$
\begin{aligned}
& \bar{A}^{\varepsilon}\left(\mathbf{u}^{\varepsilon}, \mathbf{v}^{\varepsilon}\right):=\int_{\Omega^{\varepsilon}}\left\{\lambda e_{p p}^{\varepsilon}\left(\mathbf{u}^{\varepsilon}\right) e_{q q}^{\varepsilon}\left(\mathbf{v}^{\varepsilon}\right)+2 \mu e_{i j}^{\varepsilon}\left(\mathbf{u}^{\varepsilon}\right) e_{i j}^{\varepsilon}\left(\mathbf{v}^{\varepsilon}\right)\right\} d x^{\varepsilon} \\
& \bar{L}^{\varepsilon}\left(\mathbf{v}^{\varepsilon}\right):=\int_{\Omega^{\varepsilon}} \mu_{0}\left\{M_{j}^{\varepsilon} \partial_{j}^{\varepsilon} H_{i}^{\varepsilon} v_{i}^{\varepsilon}-M_{i}^{\varepsilon} H_{j}^{\varepsilon} e_{i j}^{\varepsilon}\left(\mathbf{v}^{\varepsilon}\right)\right\} d x^{\varepsilon}+\int_{\hat{\Gamma}^{\varepsilon}} \frac{1}{2} \mu_{0}\left(M_{n}^{\varepsilon}\right)^{2} n_{i}^{\varepsilon} v_{i}^{\varepsilon} d \Gamma^{\varepsilon} .
\end{aligned}
$$

Since the bilinear form $\bar{A}^{\varepsilon}(\cdot, \cdot)$ is $V\left(\Omega^{\varepsilon}\right)$-coercive, in order to apply the Lax-Milgram's lemma, we have only to prove that the linear form $\bar{L}^{\varepsilon}(\cdot)$ is continuous on $V\left(\Omega^{\varepsilon}\right)$. For this when $1<p<+\infty$ we denote $W_{p}^{1}\left(\Omega^{\varepsilon}\right)$ the Banach space of $v \in L^{p}\left(\Omega^{\varepsilon}\right)$ whose first order derivatives (in the distribution sense) also belong to $L^{p}\left(\Omega^{\varepsilon}\right)$. The continuity of the linear form $\bar{L}^{\varepsilon}(\cdot)$ is the object of the following lemma.

Lemma 3.1 Let us assume:

$$
M_{i}^{\varepsilon}, H_{i}^{\varepsilon} \in W_{12 / 5}^{1}\left(\Omega^{\varepsilon}\right)
$$

then $\bar{L}^{\varepsilon}(\cdot)$ is continuous on $V\left(\Omega^{\varepsilon}\right)$. 
Proof. (i) Since $v_{i}^{\varepsilon} \in H^{1}\left(\Omega^{\varepsilon}\right)$, thanks to the Sobolev imbedding theorem, (see e.g. Nečas [11], Chap. 2, Theor. 3.4) we obtain that $v_{i}^{\varepsilon} \in L^{6}\left(\Omega^{\varepsilon}\right)$ and that $f_{i}^{m, \varepsilon} \in L^{6 / 5}\left(\Omega^{\varepsilon}\right)$; hence, by means of Hölder's inequality, we can infer that

$$
\left|\int_{\Omega^{\varepsilon}}\left\{\mu_{0} M_{j}^{\varepsilon} \partial_{j}^{\varepsilon} H_{i}^{\varepsilon} v_{i}^{\varepsilon}\right\} d x^{\varepsilon}\right| \leqslant C\left\|v_{i}\right\|_{1, \Omega^{\varepsilon}}
$$

(ii) Since $M_{i}^{\varepsilon}, H_{i}^{\varepsilon} \in W_{12 / 5}^{1}\left(\Omega^{\varepsilon}\right)$, thanks to the Sobolev imbedding theorem, we get that $M_{i}^{\varepsilon}, H_{i}^{\varepsilon} \in L^{12}\left(\Omega^{\varepsilon}\right) \subset L^{4}\left(\Omega^{\varepsilon}\right)$ and, thus, $M_{i}^{\varepsilon} H_{i}^{\varepsilon} \in L^{2}\left(\Omega^{\varepsilon}\right)$. It then follows from Korn's inequality

$$
\left|\int_{\Omega^{\varepsilon}}\left\{\mu_{0} M_{i}^{\varepsilon} H_{j}^{\varepsilon} e_{i j}^{\varepsilon}\left(\mathbf{v}^{\varepsilon}\right)\right\} d x^{\varepsilon}\right| \leqslant C\left\|e_{i j}^{\varepsilon}\left(\mathbf{v}^{\varepsilon}\right)\right\|_{L^{2}\left(\Omega^{\varepsilon}\right)} \leqslant C\|\mathbf{v}\|_{V\left(\Omega^{\varepsilon}\right)}
$$

(iii) By virtue of a trace imbedding theorem (see e.g. Nečas [11], Chap. 2, Theor. 4.2), we have that $\left.M_{i}^{\varepsilon}\right|_{\partial \Omega^{\varepsilon}} \in L^{8}\left(\partial \Omega^{\varepsilon}\right)$. Besides, being $\mathbf{v} \in V\left(\Omega^{\varepsilon}\right)$, then the same trace imbedding theorem imply that $\left.v_{i}\right|_{\partial \Omega^{\varepsilon}} \in L^{4}\left(\partial \Omega^{\varepsilon}\right)$, and so

$$
\left|\int_{\hat{\Gamma}^{\varepsilon}} \frac{1}{2} \mu_{0}\left(M_{n}^{\varepsilon}\right)^{2} n_{i}^{\varepsilon} v_{i}^{\varepsilon} d \Gamma^{\varepsilon}\right| \leqslant C\left\|\left(M_{n}^{\varepsilon}\right)^{2}\right\|_{L^{4}\left(\partial \Omega^{\varepsilon}\right)}\left\|v_{i}^{\varepsilon}\right\|_{L^{4}\left(\partial \Omega^{\varepsilon}\right)} \leqslant C\|\mathbf{v}\|_{V\left(\Omega^{\varepsilon}\right)}
$$

Collecting (3.4), (3.5) and (3.6), we obtain the desired result.

Thanks to the $V\left(\Omega^{\varepsilon}\right)$-coercivity of the bilinear form $\bar{A}^{\varepsilon}(\cdot, \cdot)$ and the continuity of the linear form $\bar{L}^{\varepsilon}(\cdot)$ we deduce, using the Lax-Milgram's lemma, that the variational problem (3.1) admits one and only one solution.

\section{The asymptotic expansion}

In order to perform an asymptotic analysis, we need to transform problem (3.1), posed on a variable domain $\Omega^{\varepsilon}$, onto a problem posed on a fixed domain $\Omega$ (independent of $\varepsilon$ ). We suppose that the thickness of the plate $h^{\varepsilon}$ depends linearly on $\varepsilon$, so that $h^{\varepsilon}=\varepsilon h$. Accordingly, we let

$$
\begin{aligned}
& \Omega:=\omega \times(-h, h), \\
& \Gamma_{0}:=\gamma_{0} \times(-h, h), \quad \Gamma_{1}:=\gamma_{1} \times(-h, h), \\
& \Gamma_{ \pm}:=\omega \times\{ \pm h\}, \quad \widehat{\Gamma}:=\Gamma_{ \pm} \cup \Gamma_{1} .
\end{aligned}
$$

and we define the following change of variables (see [2]):

$$
\pi^{\varepsilon}: x \equiv\left(\tilde{x}, x_{3}\right) \in \bar{\Omega} \mapsto x^{\varepsilon} \equiv\left(\tilde{x}, \varepsilon x_{3}\right) \in \bar{\Omega}^{\varepsilon} \text {, with } \tilde{x}=\left(x_{\alpha}\right) .
$$

By using the bijection $\pi^{\varepsilon}$, one has $\partial_{\alpha}^{\varepsilon}=\partial_{\alpha}$ and $\partial_{3}^{\varepsilon}=\frac{1}{\varepsilon} \partial_{3}$. Moreover, we define the following functional spaces

$$
V(\Omega):=\left\{\mathbf{v}=\left(v_{i}\right) \in H^{1}\left(\Omega ; \mathbb{R}^{3}\right) ; \mathbf{v}=\mathbf{0} \text { on } \Gamma_{0}\right\}, \quad W_{12 / 5}^{1}(\Omega) .
$$

In order to write the expression of the scaled problem, we need first to make some assumptions on the data which will define their dependences with respect to the small parameter $\varepsilon$. In our case, the only external data are represented by the magnetic field $\left(B_{i}^{\varepsilon}\right)$, the magnetic intensity field $\left(H_{i}^{\varepsilon}\right)$ and the magnetization field $\left(M_{i}^{\varepsilon}\right)$. By virtue of the constitutive equations $B_{i}^{\varepsilon}=\mu_{0} \mu_{r} H_{i}^{\varepsilon}$ 
and $M_{i}^{\varepsilon}=\chi H_{i}^{\varepsilon}$, we assume that $B_{i}^{\varepsilon}, H_{i}^{\varepsilon}$ and $M_{i}^{\varepsilon}$ will share the same dependence on $\varepsilon$. The scaling of $B_{i}^{\varepsilon}$ must reflect the fact that the magnetic field is a solenoidal field, meaning that $\partial_{i}^{\varepsilon} B_{i}^{\varepsilon}=0$ in $\Omega^{\varepsilon}$. This property must be satisfied also on the fixed domain $\Omega$. Let us suppose that

$$
B_{\alpha}^{\varepsilon}\left(x^{\varepsilon}\right)=\varepsilon^{q} B_{\alpha}(x), \quad B_{3}^{\varepsilon}\left(x^{\varepsilon}\right)=\varepsilon^{p} B_{3}(x), \quad x \in \Omega,
$$

with $B_{i}$ independent of $\varepsilon$. By applying the change of variables $\pi^{\varepsilon}$, we can write that the scaled divergence vanishes in $\Omega$, so that $\varepsilon^{q} \partial_{\alpha} B_{\alpha}+\varepsilon^{p-1} \partial_{3} B_{3}=0$ in $\Omega$. In order to guarantee the consistency of this equation, we ask that $p=q+1$, finding a relation between the two exponents $p$ and $q$. In the sequel, we choose $q=0$ and, hence,

$$
\begin{array}{lll}
B_{\alpha}^{\varepsilon}\left(x^{\varepsilon}\right)=B_{\alpha}(x), & B_{3}^{\varepsilon}\left(x^{\varepsilon}\right)=\varepsilon B_{3}(x), & x \in \Omega, \\
H_{\alpha}^{\varepsilon}\left(x^{\varepsilon}\right)=H_{\alpha}(x), & H_{3}^{\varepsilon}\left(x^{\varepsilon}\right)=\varepsilon H_{3}(x), & x \in \Omega, \\
M_{\alpha}^{\varepsilon}\left(x^{\varepsilon}\right)=M_{\alpha}(x), & M_{3}^{\varepsilon}\left(x^{\varepsilon}\right)=\varepsilon M_{3}(x), & x \in \Omega .
\end{array}
$$

With the unknown displacement field $\mathbf{u}^{\varepsilon}$, we associate the scaled unknown displacement field $\mathbf{u}(\varepsilon)$ defined by

$$
\begin{array}{ll}
u_{\alpha}^{\varepsilon}\left(x^{\varepsilon}\right)=u_{\alpha}(\varepsilon)(x) & \text { for all } x^{\varepsilon}=\pi^{\varepsilon} x \in \bar{\Omega}^{\varepsilon}, \\
u_{3}^{\varepsilon}\left(x^{\varepsilon}\right)=\frac{1}{\varepsilon} u_{3}(\varepsilon)(x) & \text { for all } x^{\varepsilon}=\pi^{\varepsilon} x \in \bar{\Omega}^{\varepsilon} .
\end{array}
$$

We likewise associate with any test function $\mathbf{v}^{\varepsilon}$, the scaled test function $\mathbf{v}$, defined by the scalings:

$$
\begin{array}{ll}
v_{\alpha}^{\varepsilon}\left(x^{\varepsilon}\right)=v_{\alpha}(x) & \text { for all } x^{\varepsilon}=\pi^{\varepsilon} x \in \bar{\Omega}^{\varepsilon}, \\
v_{3}^{\varepsilon}\left(x^{\varepsilon}\right)=\frac{1}{\varepsilon} v_{3}(x) & \text { for all } x^{\varepsilon}=\pi^{\varepsilon} x \in \bar{\Omega}^{\varepsilon} .
\end{array}
$$

According to the previous hypothesis, problem (3.1) can be reformulated on a fixed domain $\Omega$ independent of $\varepsilon$. Thus we obtain the following scaled variational problem:

$$
\left\{\begin{array}{l}
\text { Find } \mathbf{u}(\varepsilon)=\left(u_{i}(\varepsilon)\right) \in V(\Omega) \text { such that } \\
A(\mathbf{u}(\varepsilon), \mathbf{v})=L(\mathbf{v}), \text { for all } \mathbf{v}=\left(v_{i}\right) \in V(\Omega),
\end{array}\right.
$$

where the scaled bilinear form $A(\cdot, \cdot)$ and the scaled linear form $L(\cdot)$ are, respectively, defined by

$$
A(\mathbf{u}(\varepsilon), \mathbf{v}):=\frac{1}{\varepsilon^{4}} a_{-4}(\mathbf{u}(\varepsilon), \mathbf{v})+\frac{1}{\varepsilon^{2}} a_{-2}(\mathbf{u}(\varepsilon), \mathbf{v})+a_{0}(\mathbf{u}(\varepsilon), \mathbf{v})
$$

with

$$
\begin{aligned}
& a_{-4}(\mathbf{u}(\varepsilon), \mathbf{v}):=\int_{\Omega}(\lambda+2 \mu) e_{33}(\mathbf{u}(\varepsilon)) e_{33}(\mathbf{v}) d x \\
& a_{-2}(\mathbf{u}(\varepsilon), \mathbf{v}):=\int_{\Omega}\left\{4 \mu e_{\alpha 3}(\mathbf{u}(\varepsilon)) e_{\alpha 3}(\mathbf{v})+\lambda e_{\sigma \sigma}(\mathbf{u}(\varepsilon)) e_{33}(\mathbf{v})+\lambda e_{33}(\mathbf{u}(\varepsilon)) e_{\sigma \sigma}(\mathbf{v})\right\} d x \\
& a_{0}(\mathbf{u}(\varepsilon), \mathbf{v}):=\int_{\Omega}\left\{\lambda e_{\sigma \sigma}(\mathbf{u}(\varepsilon)) e_{\tau \tau}(\mathbf{v})+2 \mu e_{\alpha \beta}(\mathbf{u}(\varepsilon)) e_{\alpha \beta}(\mathbf{v})+\mu_{o} M_{i} H_{j} e_{i j}(\mathbf{v})\right\} d x \\
& L(\mathbf{v}):=\int_{\Omega} \mu_{0} M_{j} \partial_{j} H_{i} v_{i} d x+\frac{1}{2} \int_{\Gamma_{+}} \mu_{0}\left(M_{3}^{+}\right)^{2} v_{3}^{+} d \Gamma-\frac{1}{2} \int_{\Gamma_{-}} \mu_{0}\left(M_{3}^{-}\right)^{2} v_{3}^{-} d \Gamma+ \\
& \quad+\frac{1}{2} \int_{\Gamma_{1}} \mu_{0}\left(M_{\beta} n_{\beta}\right)^{2} n_{\alpha} v_{\alpha} d \Gamma
\end{aligned}
$$

where $\phi^{ \pm}:=\phi(\tilde{x}, \pm h)$ denotes the restriction of $\phi$ on $\Gamma_{ \pm}$. Since $H_{i}, M_{i} \in W_{12 / 5}^{1}(\Omega)$, thanks to lemma 3.1 and Lax-Milgram's lemma, we can prove that the scaled problem admits one and only one solution. 
We are now in position to perform an asymptotic analysis of the scaled problem (4.1). Since the scaled problem (4.1) has a polynomial structure with respect to the small parameter $\varepsilon$, we can look for the solution of the problem as a formal series of powers of $\varepsilon$ :

$$
\mathbf{u}(\varepsilon)=\mathbf{u}^{0}+\varepsilon^{2} \mathbf{u}^{2}+\varepsilon^{4} \mathbf{u}^{4}+\ldots
$$

Hence, by substituting expressions (4.2) in (4.1) and by identifying the terms with identical power of $\varepsilon$, we can write the following sequence of variational subproblems:

$$
\begin{array}{ll}
\mathcal{P}_{-4}: & a_{-4}\left(\mathbf{u}^{0}, \mathbf{v}\right)=0 \\
\mathcal{P}_{-2}: & a_{-4}\left(\mathbf{u}^{2}, \mathbf{v}\right)+a_{-2}\left(\mathbf{u}^{0}, \mathbf{v}\right)=0 \\
\mathcal{P}_{0}: & a_{-4}\left(\mathbf{u}^{4}, \mathbf{v}\right)+a_{-2}\left(\mathbf{u}^{2}, \mathbf{v}\right)+a_{0}\left(\mathbf{u}^{0}, \mathbf{v}\right)=L(\mathbf{v}) \\
\vdots &
\end{array}
$$

By solving the above variational problems, we can characterize the leading term of the asymptotic expansion $\mathbf{u}^{0}$, the so-called the limit displacement field, and its associated limit problem.

\section{The limit problem}

We define the usual functional space of Kirchhoff-Love admissible displacements:

$$
V_{K L}(\Omega):=\left\{\mathbf{v} \in V(\Omega) ; e_{i 3}(\mathbf{v})=0\right\},
$$

and

$$
\begin{aligned}
& V_{H}(\omega):=\left\{\mathbf{v}_{H}=\left(v_{\alpha}\right) \in H^{1}\left(\omega ; \mathbb{R}^{2}\right) ; \mathbf{v}_{H}=\mathbf{0} \text { on } \gamma_{0}\right\}, \\
& V_{3}(\omega):=\left\{v_{3} \in H^{2}(\omega) ; v_{3}=0 \text { and } \partial_{\nu} v_{3}=0 \text { on } \gamma_{0}\right\},
\end{aligned}
$$

where $\boldsymbol{\nu}=\left(\nu_{\alpha}\right)$ is the outer unit normal vector to $\gamma$.

Theorem 5.1 a) The leading term $\mathbf{u}^{0}$ of the asymptotic expansion (4.2) satisfies the following variational problem:

$$
\left\{\begin{array}{l}
\text { Find } \mathbf{u}^{0} \in V_{K L}(\Omega) \text { such that } \\
\mathcal{A}\left(\mathbf{u}^{0}, \mathbf{v}\right)=L(\mathbf{v}) \text { for all } \mathbf{v} \in V_{K L}(\Omega)
\end{array}\right.
$$

where

$$
\mathcal{A}\left(\mathbf{u}^{0}, \mathbf{v}\right):=\int_{\Omega}\left\{\frac{2 \mu \lambda}{\lambda+2 \mu} e_{\sigma \sigma}\left(\mathbf{u}^{0}\right) e_{\tau \tau}(\mathbf{v})+2 \mu e_{\alpha \beta}\left(\mathbf{u}^{0}\right) e_{\alpha \beta}(\mathbf{v})+\mu_{0} M_{\alpha} H_{\beta} e_{\alpha \beta}(\mathbf{v})\right\} d x .
$$

b) The sequence $\{\mathbf{u}(\varepsilon)\}_{\varepsilon>0}$ strongly converges in $H^{1}\left(\Omega ; \mathbb{R}^{3}\right)$ to $\mathbf{u}^{0}$, the solution of the limit problem (5.1).

Proof. The proof is straightforward, following the approach by [2].

Let us focus our attention to the expression of the magnetic force work $L(\cdot)$ of the limit problem. By choosing a test function $\mathbf{v} \in V_{K L}(\Omega)$, namely $v_{\alpha}\left(\tilde{x}, x_{3}\right):=\eta_{\alpha}(\tilde{x})-x_{3} \partial_{\alpha} \eta_{3}(\tilde{x})$ and $v_{3}\left(\tilde{x}, x_{3}\right):=\eta_{3}(\tilde{x})$, with $\eta_{\alpha} \in H^{1}(\omega)$ and $\eta_{3} \in H^{2}(\omega)$, after an integration along $x_{3}$ and by 
applying the Gauss-Green's formula, we get that

$$
\begin{aligned}
L(\boldsymbol{\eta})= & \int_{\Omega} \mu_{0}\left\{\left(M_{3} \partial_{3} H_{3}+M_{\alpha} \partial_{\alpha} H_{3}\right) \eta_{3}+\left(M_{\beta} \partial_{\beta} H_{\alpha}+M_{3} \partial_{3} H_{\alpha}\right)\left(\eta_{\alpha}-x_{3} \partial_{\alpha} \eta_{3}\right)\right\} d x+ \\
& +\frac{1}{2} \int_{\Gamma_{+}} \mu_{0}\left(M_{3}^{+}\right)^{2} \eta_{3} d \Gamma-\frac{1}{2} \int_{\Gamma_{-}} \mu_{0}\left(M_{3}^{-}\right)^{2} \eta_{3} d \Gamma+\frac{1}{2} \int_{\Gamma_{1}} \mu_{0}\left(M_{\beta} n_{\beta}\right)^{2} n_{\alpha}\left(\eta_{\alpha}-x_{3} \partial_{\alpha} \eta_{3}\right) d \Gamma= \\
& =\int_{\omega} \tilde{f}_{i}^{m} \eta_{i} d \tilde{x}+\int_{\gamma_{1}} \tilde{g}_{i}^{m} \eta_{i} d \tilde{x}+\int_{\gamma_{1}} \tilde{h}_{3}^{m} \partial_{\nu} \eta_{3} d \tilde{x} .
\end{aligned}
$$

where the reduced magnetic forces $\tilde{f}_{i}^{m}, \tilde{g}_{i}^{m}$ and $\tilde{h}_{3}^{m}$ have the following form

$$
\begin{aligned}
\tilde{f}_{\alpha}^{m} & :=\mu_{0} \chi\left\langle H_{i} \partial_{i} H_{\alpha}\right\rangle=\mu_{0}\left\langle f_{\alpha}^{m}\right\rangle, \\
\tilde{f}_{3}^{m} & :=\mu_{o} \chi\left\{\frac{1}{2}(\chi+1)\left(\left(H_{3}^{+}\right)^{2}-\left(H_{3}^{-}\right)^{2}\right)+\left\langle H_{\beta} \partial_{\beta} H_{3}\right\rangle+\partial_{\alpha}\left\langle\left\langle H_{i} \partial_{i} H_{\alpha}\right\rangle\right\rangle\right\}= \\
& =\mu_{0} \chi\left\{\frac{1}{2}(\chi+1)\left(\left(H_{3}^{+}\right)^{2}-\left(H_{3}^{-}\right)^{2}\right)+\left\langle H_{\beta} \partial_{\beta} H_{3}\right\rangle\right\}+\mu_{0}\left\langle\left\langle\partial_{\alpha} f_{\alpha}^{m}\right\rangle\right\rangle, \\
\tilde{g}_{\alpha}^{m} & :=\frac{1}{2} \mu_{0} \chi^{2}\left\langle H_{\nu}^{2}\right\rangle \nu_{\alpha}, \\
\tilde{g}_{3}^{m} & :=-\mu_{0} \chi\left\langle\left\langle H_{i} \partial_{i} H_{\alpha}\right\rangle\right\rangle \nu_{\alpha}=-\mu_{0}\left\langle\left\langle f_{\alpha}^{m}\right\rangle\right\rangle \nu_{\alpha}, \\
\tilde{h}_{3}^{m} & :=-\frac{1}{2} \mu_{0} \chi^{2}\left\langle\left\langle H_{\nu}^{2}\right\rangle\right\rangle,
\end{aligned}
$$

where $H_{\nu}:=H_{\alpha} \nu_{\alpha}$, and

$$
\langle\phi\rangle(\tilde{x}):=\int_{-h}^{h} \phi\left(\tilde{x}, x_{3}\right) d x_{3}, \quad\langle\langle\phi\rangle\rangle(\tilde{x}):=\int_{-h}^{h} x_{3} \phi\left(\tilde{x}, x_{3}\right) d x_{3} .
$$

It easy to verify that if the induced magnetic intensity field is normal to the middle plane of the plate, with $H_{\alpha}=0$, the form of the limit magnetic force acting on a plate depends just on the jump of the square of magnetic intensities evaluated at the top and bottom faces of the plate. Indeed, since $\tilde{f}_{\alpha}^{m}=\tilde{g}_{\alpha}^{m}=\tilde{h}_{3}^{m}=0$, one has

$$
\tilde{f}_{3}^{m}:=\frac{1}{2} \mu_{0} \chi(1+\chi)\left\{\left(H_{3}^{+}\right)^{2}-\left(H_{3}^{-}\right)^{2}\right\} \approx \frac{1}{2} \mu_{0} \chi^{2}\left\{\left(H_{3}^{+}\right)^{2}-\left(H_{3}^{-}\right)^{2}\right\},
$$

$\chi$ is very large for soft ferromagnetic materials. Equation (5.3) is analogue to the one presented in [16] and it can be considered as a mathematical justification of the magnetic force acting on a plate, which is usually employed in magnetic instability problems.

The limit problem (5.1) can be decoupled into a membrane and a bending problem, by virtue of the Kirchhoff-Love limit displacement field. The membrane problem reads as follows:

$$
\left\{\begin{array}{l}
\text { Find } \mathbf{u}_{H}^{0}=\left(u_{\alpha}^{0}\right) \in V_{H}(\omega) \text { such that } \\
\int_{\omega} n_{\alpha \beta}\left(\mathbf{u}_{H}^{0}\right) e_{\alpha \beta}\left(\boldsymbol{\eta}_{H}\right) d \tilde{x}=\int_{\omega} \tilde{f}_{\alpha}^{m} \eta_{\alpha} d \tilde{x}+\int_{\gamma_{1}} \tilde{g}_{\alpha}^{m} \eta_{\alpha} d \tilde{x} \text { for all } \boldsymbol{\eta}_{H}=\left(\eta_{\alpha}\right) \in V_{H}(\omega),
\end{array}\right.
$$

where

$$
n_{\alpha \beta}\left(\mathbf{u}_{H}^{0}\right):=\frac{4 h \lambda \mu}{\lambda+2 \mu} e_{\sigma \sigma}\left(\mathbf{u}_{H}^{0}\right) \delta_{\alpha \beta}+4 h \mu e_{\alpha \beta}\left(\mathbf{u}_{H}^{0}\right)+\mu_{0}\left\langle M_{\alpha} H_{\beta}\right\rangle,
$$

represents the ferromagnetic membrane stress tensor. After an integration by parts, we find 
that the membrane displacements $u_{\alpha}^{0}$ solve the following membrane differential problem:

$$
\begin{cases}\text { Field equation: } & \\ -\partial_{\beta} n_{\alpha \beta}=\tilde{f}_{\alpha}^{m} & \text { in } \omega, \\ \text { Boundary conditions: } & \\ n_{\alpha \beta} \nu_{\beta}=\tilde{g}_{\alpha}^{m} & \text { on } \gamma_{1}, \\ u_{\alpha}=0 & \text { on } \gamma_{0} .\end{cases}
$$

The bending problem takes the following form

$$
\left\{\begin{array}{l}
\text { Find } u_{3}^{0} \in V_{3}(\omega) \text { such that } \\
\int_{\omega} m_{\alpha \beta}\left(u_{3}^{0}\right) \partial_{\alpha \beta} \eta_{3} d \tilde{x}=\int_{\omega} \tilde{f}_{3}^{m} \eta_{3} d \tilde{x}+\int_{\gamma_{1}} \tilde{g}_{3}^{m} \eta_{3} d \tilde{x}+\int_{\gamma_{1}} \tilde{h}_{3}^{m} \partial_{\nu} \eta_{3} d \tilde{x} \text { for all } \eta_{3} \in V_{3}(\omega),
\end{array}\right.
$$

where

$$
m_{\alpha \beta}\left(u_{3}^{0}\right):=\frac{4 h^{3} \lambda \mu}{3(\lambda+2 \mu)} \Delta u_{3}^{0} \delta_{\alpha \beta}+\frac{4 h^{3} \mu}{3} \partial_{\alpha \beta} u_{3}^{0}-\mu_{0}\left\langle\left\langle M_{\alpha} H_{\beta}\right\rangle\right\rangle, \quad \Delta:=\partial_{\sigma \sigma}
$$

represents the ferromagnetic moment stress tensor. After an integration by parts, we find that the transversal displacement $u_{3}^{0}$ solves the following bending differential problem:

$$
\begin{cases}\text { Field equation: } & \\ \partial_{\alpha \beta} m_{\alpha \beta}=\frac{2 h^{3}}{3} \frac{\lambda+\mu}{\lambda+2 \mu} \Delta \Delta u_{3}^{0}-\mu_{0} \partial_{\alpha \beta}\left\langle\left\langle M_{\alpha} H_{\beta}\right\rangle\right\rangle=\tilde{f}_{3}^{m} & \text { in } \omega, \\ \text { Boundary conditions: } & \text { on } \gamma_{1}, \\ \partial_{\alpha} m_{\alpha \beta} \nu_{\beta}+\partial_{\tau}\left(m_{\alpha \beta} \nu_{\alpha} \tau_{\beta}\right)=\tilde{g}_{3}^{m} & \text { on } \gamma_{1}, \\ m_{\alpha \beta} \nu_{\alpha} \nu_{\beta}=\tilde{h}_{3}^{m} & \text { on } \gamma_{0}, \\ u_{3}=\partial_{\nu} u_{3}=0 & \end{cases}
$$

where $\boldsymbol{\tau}=\left(-\nu_{2}, \nu_{1}\right)$ represents the unit tangent vector to $\gamma$.

Considering the case of an induced magnetic intensity field, normal to the middle plane of the plate, with $H_{\alpha}=0, n_{\alpha \beta}$ and $m_{\alpha \beta}$ reduce to the classical elastic membrane stress tensor and moment stress tensor. Besides, since $\tilde{f}_{\alpha}^{m}=\tilde{g}_{\alpha}^{m}=0$, we can infer that the membrane problem admits the only zero solution, so that $n_{\alpha \beta}=0$, and thus, in this case, the plate equilibrium problem takes just into account the bending behavior.

\section{Concluding remarks}

In this work we derive a model of a soft ferromagnetic isotropic linear plate by means of an asymptotic analysis. In the absence of mechanical loading, thanks to the particular scaling of the magnetic charges, we obtain a complex expression of the reduced magnetic forces acting on the plate. The problem can be decoupled as usual in a membrane problem and in a flexural problem. It is important to notice that in the simple case in which the magnetic charges are normal to the middle plane of the plate, we formally obtain an expression of the magnetic force (5.3), acting on the plate, which is equivalent to the one used in classical literature, see, e.g., $[12,15,16]$. Moreover, by virtue of the strong convergence result, we also give a mathematical justification to the limit model. 
The present work represents a first step on the asymptotic modeling of soft ferromagnetic plates. Indeed, we do not consider the coupling between the mechanical and magnetic behaviors within the equations of magnetostatics. We assume the magnetic charges as external loads without investigating the asymptotic behavior of the magnetostatic equations for what concerns with the magnetic field, the magnetic intensity and the magnetization. Therefore, the limit problem becomes linear and we cannot see, at first glance, the so-called magnetic buckling phenomenon.

Acknowledgement We thank K. Danas and N. Triantafyllidis for many useful discussions on magnetoelastic materials.

\section{References}

[1] Brown W. F. Jr, Magnetoelastic Interactions, Springer-Verlag, Berlin Heidelberg New York, 1966.

[2] Ciarlet, P. G., Mathematical Elasticity, vol. II, Theory of Plates, North-Holland, Amsterdam, 1997.

[3] Danas, K., Triantafyllidis, N., Instability of a magnetoelastic layer resting on a non-magnetic substrate. $J$. Mech. Phys. Solids 69,2014, 67 C 83

[4] Dorfmann A., Ogden R. W., Nonlinear magnetoelastic deformations of elastomers, Acta Mechanica, 167, 2004, 13-28

[5] Kankanala, S. V., Triantafyllidis, N., Magnetoelastic buckling of a rectangular block in plane strain, J. Mech. Phys. Solids, 56 2008, 1147-1169

[6] Maugin, G.A., Eringen, A.C., Deformable magnetically saturated media I. Field equations. J. Math. Phys, 13, 1972, 143-155.

[7] Maugin, G. A., Goudjo, C., The equations of soft ferro-magnetic elastic plates, Int. J. Solid Struct., 8,1982, 889-912.

[8] Maugin, G. A., Continuum Mechanics of Electromagnetic Solids, North-Holland, Amsterdam, 1988.

[9] Moon, E. C., Magneto-solid mechanics, Wiley, New York, 1984.

[10] Miya, K., Hara, K., Someya, K., Experimental and theoretical study on magnetoelastic buckling of a ferromagnetic cantilevered beamCplate. J. Appl. Mech. 45, 1978, 355-360.

[11] Nečas, J., Direct Methods in the Theory of Elliptic Equations, Springer-Verlag, Berlin Heidelberg, 2012, Originally published 1967 in French by Academia, Praha, and Masson et Cie, Editeurs, Paris with the title Les méthodes directes en théorie des équations elliptiques.

[12] Pao, Y. H., Yeh, C. S. , A Linear Theory for Soft Ferromagnetic Elastic Solids, Int. J. Engng, 11, 1973, $415-436$.

[13] Tiersten, H.F., Coupled magnetomechanical equations for magnetically saturated insulators. J. Math. Phys, 5,1964, 1298-1318

[14] Truesdell, C., Toupin, R., The classical field theories. In: Flügge, S. (Ed.), Handbuch der Physik, Vol. III/I. Principles of Classical Mechanics and Field Theories, 226-790, Springer, Berlin, 1960

[15] Zhou, Y. H. and Zheng, X. J., A theoretical model of magnetoelastic buckling for soft ferromagnetic thin plates, Acta Mechanica Sinica (English Edition), 12, 1996, 213-224

[16] Zhou, Y. H., Zheng, X., General expression of magnetic force for soft ferromagnetic plates in complex magnetic fields, Int. J. Engng, 35, 1997, 1405-1417

[17] Zhou, Y. H., Zheng, X., Miya K., Magnetoelastic bending and snapping of ferromagnetic plates in oblique magnetic fields, Fusion Engng. design, 30, 1995, 325-337 